Results Stroke event rates for baseline and Q20 cohorts were 3.1 and 8.4 per 1000 person years respectively. At baseline, healthier levels of three LS7 - BP, physical activity and smoking were associated with reduced risk of stroke. HRs [95\% Confidence Intervals] for intermediate and ideal (vs poor) were $0.62[0.49,0.79]$ and $0.41[0.24,0.69]$ for BP; 0.68 $[0.49,0.95]$ and $0.55[0.39,0.79]$ for physical activity; and $0.68[0.54,0.86]$ and $0.57[0.43,0.77]$ for smoking. For exposures measured at $\mathrm{Q} 20$, only $\mathrm{BP}$ maintained a protective association (HRs 0.84 [0.66, 1.06] and 0.50 [0.30, 0.84] for intermediate and ideal levels respectively). Protection from each unit increase in overall CVH scores also weakened with age. With reference to the Low-Low trajectory, all trajectories were generally associated with reduced risk. The HRs were Low-High 0.57 (0.41, 0.79); High-Low 0.85 (0.61, 1.19) and High-High $0.77(0.58,1.03)$ respectively.

Conclusion Not all components of $\mathrm{CVH}$ individually influence stroke. While the association between $\mathrm{CVH}$ and stroke weakens with age, improving overall $\mathrm{CVH}$ may bring some benefit even in later life.

\section{OP53 MOLECULAR BIOMARKERS IN PERIPARTUM CARDIOMYOPATHY: A SYSTEMATIC REVIEW AND META-ANALYSIS}

${ }^{1} S$ Cherubin*, ${ }^{2}$ T Peoples, ${ }^{3}$ J Gillard, ${ }^{4} S$ Lakhal-Littleton, ${ }^{1} J$ Kurinczuk, ${ }^{1} \mathrm{M}$ Nair. ${ }^{1}$ Nuffield Department of Population Health, University of Oxford, Nuffield Department of Population Health, Oxford, UK; ${ }^{2}$ Mailman School of Public Health, Columbia University, New York, USA; ${ }^{3}$ Medical Science Division, University of Oxford, Oxford, UK; ${ }^{4}$ Department of Physiology, Anatomy and Genetics, University of Oxford, Oxford, UK

\subsection{6/jech-2020-SSMabstracts.52}

Background Peripartum cardiomyopathy (PPCM) is a complication of pregnancy in which symptoms of heart failure with reduced ejection fraction $(<45 \%)$ develop during pregnancy or shortly after delivery. The aetiology and pathophysiology of the disease are poorly understood, but there is some evidence that PPCM is associated with a) altered levels of prolactin (PRL) cleavage products, and b) anaemia. However, the strength of the evidence has not been systematically ascertained. We conducted a systematic review and meta-analysis to: 1) assess the strength of the evidence for PRL cleavage and iron deficiency anaemia as mechanisms for PPCM, 2) identify other biomarkers associated with PPCM.

Methods The search strategy for the systematic review was a mix of automated and manual searches, and included both published and unpublished literature. We included observational studies from across the world reporting levels of laboratory biomarkers in women diagnosed with PPCM and in controls without pre-existing CVD, without any restriction of language or time-period. We assessed the risk of bias and quality of the evidence across studies using standard tools. Pooled Standardized Mean Difference (SMD) were generated using a random effects model for the difference in levels of biomarkers comparing PPCM cases to healthy controls.

Results Out of 2,425 unique research articles, 78 were selected for full text screening. We extracted 31 papers, 16 of which were included in the meta-analysis. Two papers assessed the association of PRL with PPCM and reported that PPCM cases had higher levels of total PRL. Other markers investigated in PPCM patients included inflammatory markers, markers of myocardial dysfunction, vascular markers, and micronutrients. Generally, PPCM cases had higher serum levels of CRP (SMD: 2.281, 95\% CI: 0.114; 4.448), white blood cells (SMD: 0.437, 95\% CI: 0.095; 0.778), natriuretic peptides (SMD: 3.453, 95\% CI: 2.174; 4.695), cardiac troponins (SMD: 1.108, 95\% CI: 0.690; 1.526), liver enzymes (SMD: 0.651, 95\% CI: 0.075; 1.228), and creatinine (SMD: 0.513 , 95\% CI: 0.33; 0.694), but lower levels of albumin (SMD: -0.662, 95\% CI: -0.971; 0.352), selenium (SMD: -0.744, 95\% CI: -1.485; -0.002), and haemoglobin (SMD:-0.449, 95\% CI: -0.639; -0.259). We did not find any studies that analysed the association between levels of iron markers and PPCM.

Conclusion More robust epidemiologic studies are needed to strengthen the link between PRL and PPCM, identify new molecular pathways involved in the development and progression of PPCM, and elucidate the role of iron status in the pathophysiology of the disease.

\section{OP54 ENGAGEMENT IN LEISURE ACTIVITIES AND DEMENTIA RISK IN THE ENGLISH LONGITUDINAL STUDY OF AGEING}

P Almeida-Meza*, AP Steptoe, D Cadar. Behavioural Science and Health, UCL, London, UK

\subsection{6/jech-2020-SSMabstracts.53}

Background Participation in leisure activities has been associated with a lower risk of dementia. It is unclear whether increased participation in leisure activities lowers the risk of dementia or participation in leisure activities declines during the preclinical phase of dementia. We examined the frequency of participation in leisure activities and derived cognitive-activity and social activity scales and investigated dementia incidence over 15 years of follow-up in a representative sample of the English population.

Methods Data were 12,280 participants aged 50+ from the English Longitudinal Study of Ageing, free from dementia at their baseline assessments being either wave 1 (2002-2003), 3 (2006-2007), or 4 (2008-2009), and followed up until wave 8 (2016-2017). Leisure activities were derived using a standardised questionnaire derived by Nucci et al. and grouped into the cognitive and social type of leisure activities. Cox proportional hazards regression models were used to estimate the hazard ratios (HR) of dementia in relation to the cognitive and social type of leisure activities using the age of survival as the time metric.

Results During the follow-up period, 602 participants aged 56 to 99 developed dementia. Medium (HR 0.79, 95\% CI $0.65-$ $0.94, \mathrm{p}=0.013$ ) and higher levels (HR 0.62, 95\% CI $0.46-$ $0.83, \mathrm{p}=0.002$ ) of engagement in cognitive leisure activities were associated with a lower risk of dementia by survival age in a model adjusted for sex and marital status. Further adjustment for wealth explained the association with medium level, but not with higher cognitive engagement (HR 0.72, 95\% CI 0.53-0.98, $p=0.036)$. Further subsequent adjustment for CHD, stroke, hypertension did not substantially modify these associations. An independent analysis of the engagement in the social type of leisure activities showed a similar pattern with protection for higher levels of engagement (HR 0.75, 95\% CI $0.63-0.90, p=0.002)$ in a model adjusted for sex and marital status. However, further adjustment for the overall wealth has explained this association. 
Conclusion This study showed a reduced risk of dementia for individuals with a higher level of engagement in cognitively stimulating activities such as reading, playing computer games, visiting art and museums contributing to a multifaceted level of leisure activities, that may preserve cognitive reserve until later in life and hinting to the psychosocial paradigm.

\section{Thursday 10 September Diet and Obesity}

\section{OP55 CHILDHOOD OVERWEIGHT AND OBESITY AT THE START OF PRIMARY SCHOOL: EXTERNAL VALIDATION OF PREGNANCY AND EARLY-LIFE PREDICTION MODELS}

${ }^{1} \mathrm{~N}$ Ziauddeen*, ${ }^{1} \mathrm{PJ}$ Roderick, ${ }^{2} \mathrm{G}$ Santorelli, ${ }^{2} \mathrm{~J}$ Wright, ${ }^{1,3} \mathrm{NA}$ Alwan. School of Primary Care, Population Sciences and Medical Education, University of Southampton, Southampton, UK; ${ }^{2}$ Bradford Institute for Health Research, Bradford Royal Infirmary, Bradford, UK; ${ }^{3} \mathrm{NIHR}$ Southampton Biomedical Research Centre, University of Southampton and University Hospital Southampton NHS Foundation, Southampton, UK

\subsection{6/jech-2020-SSMabstracts.54}

Background Tackling the childhood obesity epidemic can potentially be facilitated by risk-stratifying families at an earlystage to receive prevention interventions and extra support. As part of the Studying Lifecourse Obesity Predictors (SLOPE) study, we developed prediction models for childhood overweight and obesity using routinely-collected antenatal and early-life healthcare data in Hampshire, South of England. ${ }^{1}$ As a model usually performs better in the data used for its development, external validation is needed to check its performance in similar but new target population. This analysis aimed to externally validate these models using data from the Born in Bradford (BiB) cohort in the North of England.

Methods $\mathrm{BiB}$ is a longitudinal multi-ethnic birth cohort study which recruited 12,453 women (13,776 pregnancies) at around 28 weeks gestation between 2007 and 2010 in Bradford. Data from the routine National Child Measurement Programme measurement at 4-5 years in school was linked to the maternal and early-life $\mathrm{BiB}$ cohort data. The outcome was defined as body mass index (BMI) $\geq 91$ st centile at 4-5 years based on the UK clinical cut-off used to develop the SLOPE models. Maternal predictors included BMI, highest educational attainment, partnership and smoking status at booking, ethnicity and intake of folic acid supplements. Early life predictors included birthweight, gestational age, sex and weight at 1 or 2 years. Discrimination was assessed using the area under the receiver operating curve (AUC) and calibration using calibration slope (equal to one in well-calibrated model).

Results Data were available for 6292 women for the early pregnancy models and 3801 women and children for the early-life models. The AUC was comparable to the development model at all stages (early pregnancy, birth, $\sim 1$ year and $\sim 2$ years). The AUC at development was 0.66 (95\% confidence intervals (CI) 0.65 to 0.67 ) compared to 0.64 (95\% CI 0.62 to 0.66$)$ on external validation. Similarly, the AUC was 0.83 (95\% CI 0.82 to 0.84 ) at $\sim 2$ years at development and 0.81 (95\% CI 0.79 to 0.83 ) on external validation. Models were less well-calibrated on external validation ranging from 0.87 (standard error (SE) 0.04) to 0.91 (SE 0.06) across the stages compared to 0.98 (SE 0.03) to 0.99 (SE 0.01) at development.
Conclusion The SLOPE models developed for predicting childhood overweight and obesity risk performed reasonably well on external validation in a birth cohort with a different geographical location and ethnic composition. However, recalibration by updating the model intercept may be required to improve calibration in other populations.

\section{REFERENCES}

1. Ziauddeen N, Wilding S, Roderick PJ, et al. Predicting the risk of childhood overweight and obesity at 4-5 years using population-level pregnancy and earlylife healthcare data. BMC Med 2020;18:105. https://doi.org/10.1186/s12916020-01568-z

\section{OP56 WHAT IS THE LIKELIHOOD OF OVERWEIGHT OR OBESITY IN YOUNGER CHILDREN SHARING A HOUSEHOLD WITH AN OLDER CHILD WHO IS OBESE OR SEVERELY OBESE? CROSS-SECTIONAL ANALYSIS OF LINKED NATIONAL CHILD MEASUREMENT PROGRAMME DATA}

${ }^{1} \mathrm{~N}$ Firman*, ${ }^{1} \mathrm{G}$ Harper, ${ }^{1} \mathrm{Z}$ Ahmed, ${ }^{1} \mathrm{~J}$ Robson, ${ }^{1} \mathrm{~K}$ Boomla, ${ }^{2} \mathrm{D}$ Stables, ${ }^{1} \mathrm{C}$ Dezateux. ${ }^{1}$ Clinical Effectiveness Group, Institute of Population Health Sciences, Queen Mary University of London, London, UK; ${ }^{2}$ Discovery Data Service, Endeavour Health Charitable Trust, UK

\subsection{6/jech-2020-SSMabstracts.55}

Background The National Child Measurement Programme (NCMP) assesses the weight status of 4-5 and 10-11 year-old children attending state schools. We linked pseudonymised NCMP records to pseudonymised unique household identifiers derived from general practice electronic health records to investigate the likelihood of overweight or obesity in a younger household child member based on the oldest child's weight status, sex and ethnic background.

Methods We assigned an encrypted Unique Property Reference Number using a validated address-matching algorithm to addresses of patients registered in mid-2018 with all 159 GP practices in four inner east London boroughs. We identified 36,789 children (18,714 five-year-olds [9,326 girls] and 18,075 11-year-olds [8,989 girls]) with NCMP records living in 16,434 households, representing $33.8 \%$ of 48,602 registered households with two or more children who would have been eligible for NCMP measurements in the preceding five school years. We identified the oldest child in the household with a NCMP record as the reference child.

We used binary logistic regression to estimate the likelihood (Odds Ratio [OR]; 95\% confidence intervals [CI]) of at least one younger household member with NCMP-recorded overweight or obesity (BMI centile $\geq 91 \mathrm{st}$ ) according to the reference child's weight status (reference category: healthy weight), sex (male), and ethnic background (White) after adjustment for household size and composition, and borough of residence (Stata/MP 15).

Results The number of children with a NCMP record per household ranged from 2-8 (median 2, IQR 2-2) with 13,170 (80.1\%) including only two. $50.0 \%$ of 16,434 reference children were girls, with $18.2 \%, 33.5 \% 18.6 \%$ from White, South Asian or Black ethnic backgrounds respectively. 5.4\% $(n=890)$ of reference children were severely obese $(\geq 120 \%$ of the 95 th centile) and $24.0 \%(n=3,936)$ had a younger household member with NCMP-recorded overweight or obesity.

After mutual adjustment, reference children who were obese (OR 3.50; 95\% CI: 3.08,3.96), severely obese (4.29; $3.69,4.99)$ or from Black ethnic backgrounds (1.52; 